11 Han J, Wilson A, Wesley E. Delirium in the older emergency department patient: a quiet epidemic. Emerg Med Clin North Am 2010;28:611-31.

12 Young J, Inouye SK. Delirium in older people. BMJ 2007;334:842-6.

13 Han JH, Zimmerman EE, Cutler N et al. Delirium in older emergency department patients: recognition, risk factors, and psychomotor subtypes. Acad Emerg Med 2009;16:193-200.

14 van der Wardt V. Should guidance for the use of antihypertensive medication in older people with frailty be different? Age Ageing 2015;44:912-3.

15 O’Mahony D, O’Sullivan D, Byrne S et al. STOPP/START criteria for potentially inappropriate prescribing in older people: version 2 . Age Ageing 2014;44:213-8.
16 Fox C, Richardson K, Maidment ID et al. Anticholinergic medication use and cognitive impairment in the older population: the medical research council cognitive function and ageing study. J Am Geriatr Soc 2011;59:1477-83.

17 Gill TM, Gahbauer EA, Han L, Allore HG. Trajectories of disability in the last year of life. N Engl J Med 2010;362:1173-80.

Address for correspondence: Dr Simon Conroy, Geriatric Medicine, Leicester Royal Infirmary, Infirmary Square, Leicester LE1 5WW, UK.

Email:spc3@le.ac.uk

\title{
Making difficult decisions with older patients on medical wards 洸结
}

\author{
Authors: Bushra Khizar ${ }^{\mathrm{A}}$ and Rowan $\mathrm{H}$ Harwood $^{\mathrm{B}}$
}

Decision making with older people can be difficult because of medical complexity, uncertainty (about prognosis, treatment effectiveness and priorities), difficulties brought by cognitive and communication impairment and the multiple family and other stakeholders who may need to be involved. The usual approach, based on balancing benefits and burdens of a treatment, and then deciding on the basis of autonomy (or best interests for someone lacking mental capacity), within the constraints of resources and equity, remains valid, but is often inadequate. In addition, approaches relying on optimal communication and relationship building and professional virtues are important. Older people vary in their medical status, views and preferences more than younger people and these variations must be sought and accommodated, using a shared decision-making approach. This includes adapting to the increasing numbers of people from different cultures.

\section{How do we decide?}

Scientific knowledge, the law, professional regulation, the organisations within which we work and the political and social environment all influence how we practise medicine. Quite apart from law and regulation, and sometimes in contravention to it, people have expectations about healthcare: who should be consulted over decisions and how we should approach death

Authors: ${ }^{\text {A }}$ specialist registrar, Nottingham University Hospitals NHS Trust, Nottingham, UK; ${ }^{B}$ consultant geriatrician, Nottingham University Hospitals NHS Trust, Nottingham, UK and dying. No single set of rules adequately covers all human activity, including medical practice, but ethical frameworks can help us analyse and understand difficult situations, and resolve or address them through better understanding.

\section{Key points}

Older people are medically complex, the information needed to make decisions is often uncertain and the impact of cognitive impairment can make decision making difficult

A systematic and thorough approach is required; the principlist approach of considering benefits, burdens, autonomy and justice can be applied, but principles often conflict

Open communication and relationship building with families and other stakeholders assumes greater importance

Individual variation must be identified, respected and accommodated in making decisions

Decisions at the end of life are open to the same general approach, but uncertainty in establishing prognosis and widespread overestimation of the efficacy of medical intervention can lead to over-intervention and a failure to adopt a palliative approach

KEYWORDS: Cognitive impairment, decision making, end-of-life care, ethics, older people, professional 


\section{Ethical systems}

Ethical frameworks fall broadly into four approaches: ${ }^{1}$

1 Actions may be judged by their consequences: we try to balance the possible good and bad effects of interventions.

2 Actions must respect rights and duties (deontology). Many fundamental rights are enshrined in law and conventions, such as the right to life, to have personal information kept confidential or to be consulted about medical treatment.

3 Virtues are personal characteristics, such as honesty, prudence, courage, compassion, forgiveness and generosity, that form the basis of professional practice, many religious teachings and cultural expectations.

4 Communication and relationship-based ethics suggest that the right action is the one that results from exchange of information and views from the perspectives of all stakeholders, and seeks to maintain and develop relationships.

In practice, we draw on different frameworks at different times. Sometimes they conflict.

The most important contemporary framework for medical decision making is called 'principlism'. ${ }^{2}$ It combines two approaches - consequentialism and deontology (Box 1). Together the principles allow decisions to be made in most medical situations. An intervention should produce more good than harm, or the degree of risk or burden of unwanted effects should be proportionate to, and justified by, the likely good. Autonomy - the right to self-determination - should be respected; it is expressed through the giving or withholding of consent (Box 2). Interventions incur costs, which must either be met by the individual, or collectively, in which case resources will not be available to deliver potentially more valuable alternatives. An equitable basis for allocation (or denial) of resources is required.

\section{Application with older people}

Key problems in the case of older patients include lack of mental capacity, uncertainty, complexity, communication problems, resource limitation and social expectations.

Dementia and delirium affect about half of older inpatients in acute hospitals. ${ }^{3,4}$ These (and various other conditions)

\section{Box 1. Principlism}

All of the principles must be satisfied for an action to be ethical:

Benefits: the action is likely to result in a good outcome

Burdens and risks: the action must avoid adverse or unwanted effects, risks, inconvenience, debility or these must be proportionate to, and justified by, the good that results

$>$ Autonomy: we must respect the right for an individual (with mental capacity) to make choices for themselves, including balancing likely benefits and burdens, even if the choice appears to be unwise. Autonomy is exercised through the giving or withholding of consent

> Justice: we must avoid discrimination on the basis of age, gender, race, disability or other characteristics irrelevant to the decision, and we must work equitably within limited resources
Box 2. Mental capacity, consent and best interests

Mental capacity describes the ability of an individual to make decisions. Having mental capacity requires the ability to understand, retain and use information to make a decision, and to be able to communicate that decision. Understanding should include the nature, purpose and consequences of the proposed intervention, any alternatives, adverse effects or risks, and the consequences of refusal. Capacity is decision- and time-specific.

Consent is agreement to have an examination, investigation, treatment, care or surgery. To be valid, consent must be:

$>$ capacitous (the person must understand what they are doing)

$>$ informed (the person must have enough information on which to base a decision)

$>$ uncoerced (freely given)

> ongoing (consent can be withdrawn at any time).

In the UK, a treatment may be legally given to a person who lacks mental capacity if it is in their 'best interests'. This attempts to respect autonomy by involving them in the decision if possible, and trying to identify what they would have wanted if they had capacity. The process is often uncertain. Family members or others concerned for the patient's wellbeing have a right to have their opinion heard. Best interests should take account of current and previously expressed values, preferences or beliefs. The treatment should have overall benefit when weighing the risks and burdens associated with it.

are often associated with impairment of mental capacity to make decisions (Box 2). Strictly, mental capacity should be assessed for every decision, about every examination, test, care procedure or treatment, but usually this will be informal and implicit, reserving formal assessment for important, or contentious, decisions. Assessing mental capacity in practice can be difficult, with many cases borderline or uncertain. Questions that arise include:

$>$ How much understanding must be shown?

> How long must information be retained?

$>$ How is reasoning or use of information to be demonstrated?

$>$ Which possible consequences or alternatives should be revealed?

Older people are medically complex and frequently diagnosis, prognosis and the effectiveness of interventions are uncertain. Treatment guidelines may not apply; ${ }^{5}$ a greater reliance on professional judgement is required, for example balancing likely investigation- or treatment-burden against the probability of finding treatable pathology or delivering meaningful health gain. Older patients may struggle to understand or communicate because of cognitive or sensory impairment, language, education or culture. In assessing capacity, explaining and taking consent, and assessing best interests, communication should be adapted to help the person have the best chance of success; hearing aids, glasses, translators, family or other advocates, speech and language therapists or pictorial material may all be needed.

Health and care services for older people are less wellresourced than those for other population groups. The divide 
between health and social care is arbitrary, but healthcare is free at the point of delivery while social care in the UK is means-tested. These are primarily political choices, but impact on everyday decision making - for example, when the resources required to enable a safe discharge home are not available or if a patient or family member declines to pay for necessary services.

\section{Variation between individuals}

The reduction of 'unwarranted variation' in clinical practice has become an important mechanism for improving efficiency and safety. However, implicit in the delivery of person-centred care and respecting the differing priorities, preferences and values of individuals in keeping with mental capacity law is that we accept differences. A minority of people value the preservation of life at almost all cost. Some people have strong views on feeding and hydration at the end of life. Others feel strongly that they should have control over when they die should the circumstances arise. We are bound by the law, our professional regulators and resources, but we must try to accommodate differing preferences.

The British population is multicultural. An increasing number of older people were born outside the UK. Concepts of health, illness, suffering and care mean different things in different cultures. Western culture mostly emphasises individualism and autonomy, whereas Eastern philosophies stress harmony, respect, relationships and community or group solidarity. In healthcare, these need not be antagonistic; respect for variation between people can accommodate the preferences of patients of any culture. Problems can arise where differences are not well understood, for example, that the senior male family member may be expected to assume the role of caregiver and decision maker. Any decision making can be delegated: people of all cultures often welcome family support in making difficult decisions and so long as the individual is not coerced, this is consistent with Western ethics and law. ${ }^{6}$ These matters are best openly discussed. We are also fortunate that professional colleagues from the same culture may be able to mediate if there is disagreement.

\section{Communication and relationship-based ethics}

Rigid ethical frameworks become less useful in the face of uncertainty, complexity and loss of mental capacity, when key information may be lacking (such as what an individual thinks or values) or when ethical principles conflict. In these cases, an approach that emphasises open communication, open-mindedness and a commitment to building and maintain trusting relationships can help negotiate decisions in the face of possible conflict and accusation, for example about whether an action is truly in someone's 'best interests.' ${ }^{1}$ This can involve a lot of exploration, explanation, deliberation, consensus building (and time), and is embodied in the approach of 'shared decision making.' This aims to identify the options, set out the pros and cons of each, and then work together to reach a decision. However, communication and relationship-based approaches have limitations. It is not always possible to reach agreement and it is not sufficient simply to do as the patient or family want in order to 'keep them happy'.

\section{Advance care planning}

Advance care planning allows patients to make their wishes known about treatment decisions in the future when they may not be able to do so themselves. ${ }^{8}$ It may take the form of a statement of wishes or preferences, an advance decision to refuse treatment or the appointment of a lasting power of attorney (a legal device to give a person authority to act as a proxy to make decisions). Early advance care planning is important, especially for patients living with progressive conditions likely to impair mental capacity (especially dementia).

\section{Discharge decisions}

Discharge planning is a process that aims to improve the coordination of services after discharge from hospital by considering the patient's needs in the community. Issues arise where insight is lacking about abilities and risks, or where undue help from family or professional carers is expected by the patient. This can only be managed by a multidisciplinary health team, including occupational therapists in particular, with decisions made jointly with families and community services.

'Risk enablement', or 'positive risk management', is based on the idea of balancing the benefits and risks against the negative effects of attempting to avoid risk altogether. ${ }^{9}$ Risks are identified and acknowledged, minimised or mitigated where possible, then an overt decision is made on whether to accept the residual risk or not. If the patient has capacity, this is on the basis of informed consent; if the patient does not have capacity, a best interests decision is required involving family or other carers. Crucial to this will be ascertainment of the individual's attitude to risk and the relative value placed on living at home or in a care home.

\section{The end of life}

Difficult decisions in end-of-life care are often around starting, or stopping, potentially life-prolonging treatments such as cardiopulmonary resuscitation, renal dialysis, artificial nutrition and hydration, or adopting an overtly palliative approach to care. Decisions to withhold or withdraw lifesustaining treatments are usually driven by an assessment that such treatments would be unlikely to work (ie are futile), which is often, to an extent, uncertain. Palliative care focuses on relieving distress, maintaining function, minimising investigation- or treatment-related burden and planning for deterioration or crisis.

These are open to the general principles of decision making (weighing benefits and burdens, respecting autonomy or assessing best interests, and using open communication with stakeholders to reach consensus). The issues of resuscitation and artificial nutrition and hydration are plagued by poor understanding about when they are, or are not, effective at prolonging life/bringing health gain, and ill-informed or mendacious media and political coverage. Current guidelines are sensible and measured. ${ }^{10-13}$ There is no obligation to provide a futile intervention although there is a duty to inform able patients and the families of those lacking capacity. A shared decision-making approach is required where the balance of 
benefits and burdens, or the likely wishes of the individual are uncertain.

\section{Conflicts of interest}

The authors have no conflicts of interest to declare.

\section{References}

1 Hughes J, Baldwin C. Ethical issues in dementia care: making difficult decisions. London: Jessica Kingsley Publishers, 2007.

2 Beauchamp TL, Childress JF. Principles of biomedical ethics, 7th edn. New York: Oxford University Press, 2013.

3 Jackson TA, Gladman JRF, Harwood RH et al. Challenges and opportunities in understanding dementia and delirium in the acute hospital. PLoS Med 2017;14:e1002247.

4 Goldberg SE, Whittamore K, Harwood RH et al. The prevalence of mental health problems amongst older adults admitted as an emergency to a general hospital. Age Ageing 2012;41:80-6.

5 National Institute for Health and Care Excellence. Multimorbidity: clinical assessment and management. NICE guideline No 56. London: NICE, 2016.

6 Pacquiao DF. Ethics and cultural diversity: a framework for decision making. Bioethics Forum 2001;17:12-7.

7 Coulter A, Collins A. Making shared-decision making a reality: no decision about me, without me. London: Kings Fund, 2011.
8 Royal College of Physicians of London. Advance care planning. Concise guidance to good practice series No 12. London: RCP, 2009.

9 Manthorpe J, Moriaty J. Nothing ventured, nothing gained. Risk guidance for people with dementia. London: Department of Health, 2010.

10 British Medical Association, Resuscitation Council (UK), Royal College of Nursing. Decisions relating to cardiopulmonary resuscitation, 3rd edn. London: BMA, 2016.

11 General Medical Council. Treatment and care towards the end of life: decision making. London: GMC, 2010.

12 American Geriatrics Society. American Geriatrics Society feeding tubes in advanced dementia position statement. J Am Geriatr Soc 2014;62:1590-3.

13 Royal College of Physicians and British Society of Gastroenterology. Oral feeding difficulties and dilemmas: A guide to practical care, particularly towards the end of life. London: RCP, 2010.

Address for correspondence: Professor Rowan Harwood, Queen's Medical Centre, Derby Road, Nottingham NG7 2UH, UK.

Email: rowan.harwood@nuh.nhs.uk 